\title{
A Kronecker-based Covariance Specification for Spatially Continuous Multivariate Data
}

\section{Angélica Maria Tortola Ribeiro ( $\nabla$ angelicaribeiro@utfpr.edu.br )}

Federal Technological University of Paraná https://orcid.org/0000-0003-2793-7488

\section{Paulo Justiniano Ribeiro Jr}

Federal University of Paraná

\section{Wagner Hugo Bonat}

Federal University of Paraná

\section{Research Article}

Keywords: Gaussian random fields, Covariance functions, Geostatistics, Matérn correlation model, Multivariate spatial data

Posted Date: January 7th, 2022

DOI: https://doi.org/10.21203/rs.3.rs-1162646/v1

License: (a) This work is licensed under a Creative Commons Attribution 4.0 International License. Read Full License 


\title{
A Kronecker-based covariance specification for spatially continuous multivariate data
}

\author{
Angélica Maria Tortola Ribeiro ${ }^{1,3^{*}}$, Paulo Justiniano Ribeiro \\ Junior $^{2,3}$ and Wagner Hugo Bonat ${ }^{2,3}$ \\ $1^{*}$ Academic Department of Statistics, Federal Technological \\ University of Paraná, Curitiba, 80230-901, Paraná, Brazil. \\ ${ }^{2}$ Department of Statistics, Federal University of Paraná, Curitiba, \\ 81530-000, Paraná, Brazil. \\ ${ }^{3}$ Post-Graduate Program in Numerical Methods in Engineering, \\ Federal University of Paraná, Curitiba, 81530-000, Paraná, Brazil.
}

${ }^{*}$ Corresponding author(s). E-mail(s): angelicaribeiro@utfpr.edu.br;

Contributing authors: paulojus@ufpr.br; wbonat@gmail.com;

\begin{abstract}
We propose a covariance specification for modeling spatially continuous multivariate data. This model is based on a reformulation of Kronecker's product of covariance matrices for Gaussian random fields. We illustrate the case with the Matérn function used for specifying marginal covariances. The structure holds for other choices of covariance functions with parameters varying in their usual domains, which makes the estimation process more accessible. The reduced computational time and flexible generalization for increasing number of variables, make it an attractive alternative for modelling spatially continuous data. Theoretical results for the likelihood function and the derivatives of the covariance matrix are presented. The proposed model is fitted to the literature's soil250 dataset, and adequacy measures, forecast errors and estimation times are compared with the ones obtained based on classical models. Furthermore, the model is fitted to the classic meuse dataset to illustrate the model's flexibility in a four-variate analysis. A simulation study is performed considering different parametric scenarios to evaluate the asymptotic properties of the maximum likelihood estimators. The satisfactory results, its simpler structure and the reduced estimation time make the proposed model a candidate approach for multivariate analysis of spatial data.
\end{abstract}


Keywords: Gaussian random fields, Covariance functions, Geostatistics, Matérn correlation model, Multivariate spatial data

\section{Introduction}

Multivariate random fields have been of interest from the very early days of the geostatistical literature, with an increasing number of proposed approaches as data sets became richer and the ever-increasing computational power. The specification of the covariance structure is central in the estimation and prediction process. Recent contributions include asymmetric models (Qadir et al, 2021; Alegría et al, 2018), modeling on spheres (Bevilacqua et al, 2020; Emery et al, 2019; Alegría et al, 2019; Emery and Porcu, 2019), on mapping disease (Martinez-Beneito, 2020; MacNab, 2018, 2016), on multinary problems (Teichmann et al, 2021), to name a few.

We are interested in multivariate random fields analysis, in the specific context of spatially continuous data. Possible applications cover a wide range of disciplines, such as climatology, meteorology, geophysics, among others, where spatially referenced data is usually of interest. We consider two illustrative examples, one on chemical soil properties relevant for agriculture and the other on heavy metal concentrations within a study area.

Let $\mathbf{Y}(\mathbf{s})=\left\{Y_{1}(\mathbf{s}), \ldots, Y_{p}(\mathbf{s})\right\}^{\top}$, on $\mathbb{R}^{d}, d \geq 1$, a $p$-dimensional multivariate Gaussian random field with mean vector $\boldsymbol{\mu}(\mathbf{s})=E[\mathbf{Y}(\mathbf{s})]$ and matrix-valued covariance function:

$$
\boldsymbol{\Sigma}(\mathbf{h})=\operatorname{cov}\left\{\mathbf{Y}\left(\mathbf{s}_{\mathbf{1}}\right), \mathbf{Y}\left(\mathbf{s}_{\mathbf{2}}\right)\right\}=\left[\Sigma_{i j}(\mathbf{h})\right]_{i, j=1}^{p},
$$

where $\mathbf{h}=\mathbf{s}_{\mathbf{1}}-\mathbf{s}_{\mathbf{2}} \in \mathbb{R}^{d}$ is the spatial separation vector. We consider a stationary and isotropic process (Chilès and Delfiner, 2012; Diggle and Ribeiro Jr, 2007; Gneiting, 1999) where $i=j$, the functions $\Sigma_{i i}(\mathbf{h})$ in Eq. (1) describe the spatial variability of the $i$ th process $Y_{i}(\mathbf{s})$, for $i=1, \ldots, p$, and are referred as the direct- or marginal-covariance functions (Genton and Kleiber, 2015) and, if $i \neq j$, the functions $\Sigma_{i j}(\mathbf{h})$ in Eq. (1) describe the spatial variability between the process $Y_{i}(\mathbf{s})$ and $Y_{j}(\mathbf{s})$ and are called as cross-covariance functions. An important condition for (1) is that it must meet the positive definite condition, that is, $\mathbf{a}^{\top} \mathbf{\Sigma} \mathbf{a}>0$, for any vector $\mathbf{a} \neq \mathbf{0}$.

The main goal is to propose a valid covariance specification for (1) in the case of spatially continuous multivariate data. The model, presented in Section 2, is based on the Kronecker products and it is quite flexible to handle with two or more variables. Furthermore, we present the conditions for the positive definiteness and detail the inferential process of the proposed model.

The literature on covariance functions for multivariate random fields is extensive. A careful review of the main works in the area can be found in Genton and Kleiber (2015) and Salvaña and Genton (2020). An intuitive early 
proposal and possibly the most traditional model is the linear model of corregionalization (LMC) (Goulard and Voltz, 1992; Bourgault and Marcotte, 1991; Wackernagel, 2003). The key idea for the LMC is the overlap of spatial processes in order to induce a multivariate field. This approach is widely explored, including under the Bayesian approach for inference and prediction. Finley et al (2015), Banerjee et al (2003), Gelfand et al (2004), Schmidt and Gelfand (2003) and Cecconi et al (2016) are examples where the LMC structure underlies the models.

Another popular structure considers the class of Matérn correlation functions (Matérn, 1986; Guttorp and Gneiting, 2006). For the univariate case, the Matérn class covariance model is defined as $\sigma^{2} M(\mathbf{h} \mid \nu, 1 / \phi)$, where $M(\mathbf{h} \mid \nu, 1 / \phi)=\frac{2^{1-\nu}}{\Gamma(\nu)}(|\mathbf{h}| / \phi)^{\nu} K_{\nu}(|\mathbf{h}| / \phi)$, is the Matérn spatial correlation at distance $|\mathbf{h}|, K_{\nu}$ is the modified Bessel function, $\sigma^{2}, \nu, \phi>0$ are the variance, smoothness and scale parameters, respectively. When $\nu=0.5$, the Matérn model reduces to the exponential covariance function. Gneiting et al (2010) elegantly extended this class for multivariate case considering the Matérn family for the marginal and cross-covariance functions. The authors present conditions for the parameters that lead to a valid covariance structure, the full bivariate Matérn model. For more than two variables the authors presented the parsimonious multivariate Matérn model, which considers common scale and constrained smoothness parameters. Another important specification are the separable models, which considers that the components of the multivariate random field share the same correlation structure (Bevilacqua et al, 2016a; Vallejos et al, 2020) and appears as a parsimonious modeling alternative because it allows a simplification of the more complex models. Bevilacqua and MoralesOñate (2018) and Vallejos et al (2020) present two simplified structures with Matérn correlation function for bivariate data, the bivariate separable Matérn model and the bivariate Matern model with constraints. Both models can be estimated by the Geomodels package (Bevilacqua and Morales-Oñate, 2018).

The aforementioned models are widely assessed in the geostatistical literature. Cressie (1993), Gneiting et al (2010), Goovaerts et al (1997), Porcu et al (2013), Bevilacqua et al (2016b) noticed some difficulties to handling with the LMC due, for example, to its lack of flexibility and difficulty in recovering the smoothness of latent processes. Separable models are not capable to capture the different scales and smoothness for the variables under study (Bevilacqua et al, 2016a, 2015). The bivariate Matérn model presents some restrictions in the parametric space. Vallejos et al (2020) note that variation of the colocated correlation parameter is constrained by the values of the scale and smoothness parameters, resulting in difficulties for the estimation process and parameter interpretation.

The covariance specification presented here emerges as an additional modeling alternative for multivariate random fields that can be more flexible to deal with two or more variables, as it allows the model parameters to vary freely in their usual parametric domains. With a simple construction, the computational implementation has no major difficulties for a number of variables and 
sampling location points, with a parsimonious estimation computational time, especially for small sample sizes. Furthermore, unlike the separable models, our proposal allows different marginal correlation structures, making it able to capture the structure of each variable.

The article is organized as follows. In Section 2 we present our covariance specification for multivariate spatial data and discuss the maximum likelihood estimation for model parameters. The dataset analyses are presented in Section 3. In Section 4, through a simulation study, we evaluate the asymptotic properties of the proposed model estimators. Finally, the main conclusions are summarized in Section 5. The model implementation and reported analysis are performed using the computational statistical software R (R Core Team, 2021).

\section{Model specification}

This section presents our proposed covariance specification for multivariate Gaussian random fields, which is based upon Martinez-Beneito (2013).We present the proof of its validity and discuss how to obtain the maximum likelihood estimates of the model parameters.

In Martinez's proposal, the results are presented for modeling multivariate mapping diseases problems based on Gaussian Markov random fields (GMRF), which are discretely indexed, following a Gaussian multivariate distribution with the additional restriction of conditional independence (Rue and Held, 2005).

Our proposal extends Martinez's approach to construct a covariance function for Gaussian random fields that are continuously indexed, with several applications in geostatistical problems.

We present a simple construction that allows its generalization to larger dimensions more easily. The idea is to write the cross-covariance matrix as a product of matrices that induce variability within processes and between processes, and it is built upon the Kronecker products reformulation of covariance matrices. The resulting construction will be always positive definite for any parameter values in their usual domains.

Consider a symmetric correlation matrix $\Sigma_{b}$, with dimension $p \times p$, induces a spatial correlation between the processes, while the marginal-covariance functions $\Sigma_{i i}$, for $i=1, \ldots, p$, model the variability within each process. We specify the covariance matrix for the $\mathbf{Y}$ process considering the generalized Kronecker product, presented in Martinez-Beneito (2013). Thus, for the Gaussian random fields continuously indexed, the matrix-valued covariance function is defined by:

$$
\boldsymbol{\Sigma}(\mathbf{h})=B_{\text {diag }}\left(\tilde{\boldsymbol{\Sigma}}_{11}, \tilde{\boldsymbol{\Sigma}}_{22}, \ldots, \tilde{\boldsymbol{\Sigma}}_{p p}\right)\left(\boldsymbol{\Sigma}_{b} \otimes \mathbf{I}\right) B_{\operatorname{diag}}\left(\tilde{\boldsymbol{\Sigma}}_{11}^{\top}, \tilde{\boldsymbol{\Sigma}}_{22}^{\top}, \ldots, \tilde{\boldsymbol{\Sigma}}_{p p}^{\top}\right)
$$


where, $\tilde{\boldsymbol{\Sigma}}_{i i}$ is the lower triangular matrix of the Cholesky decomposition of the matrix $\Sigma_{i i}, B_{\text {diag }}$ represents the matrix in diagonal blocks of the matrices $\tilde{\boldsymbol{\Sigma}}_{11}$, $\tilde{\boldsymbol{\Sigma}}_{22}, \ldots, \tilde{\boldsymbol{\Sigma}}_{p p}$ and $\mathbf{I}$ is the identity matrix. The structure defined in (2) is very flexible, allowing different marginal-covariance functions for $\Sigma_{i i}$ and different correlation structures for $\boldsymbol{\Sigma}_{b}$.

Without loss of generality, we will consider that the spatial correlation between the processes will be induced by the correlation matrix:

$$
\boldsymbol{\Sigma}_{b}=\left(\begin{array}{cccc}
1 & \rho_{12} & \ldots & \rho_{1 p} \\
\rho_{12} & 1 & \ldots & \rho_{2 p} \\
\ldots & \ldots & \ldots & \ldots \\
\rho_{1 p} & \rho_{2 p} & \ldots & 1
\end{array}\right)
$$

where $\rho_{i j}, i j=1, \ldots, p$, is the correlation parameter between the variables $i$ and $j$.

To quantify the variability within each process we will use, in this work, the Matérn covariance function, that is,

$$
\Sigma_{i i}(\mathbf{h})=\sigma_{i}^{2} M\left(\mathbf{h} \mid \nu_{i}, 1 / \phi_{i}\right), \quad \text { for } \quad i=1,2, \ldots, p .
$$

The structure specified by (2), (3) and (4) will be called as simpler multivariate Matérn (MatSimpler) model. It accepts different marginal behaviors and is able to handle with different smoothness and scale parameters for each variable. The simpler multivariate exponential (ExpSimpler) model is a particular case when the exponential correlation function is used. Ribeiro et al (2021) illustrates the ExpSimpler model in bivariate analysis of meteorological data.

In Theorem 2.1, below, we prove the validity of our covariance specification for modeling multivariate spatial data.

Theorem 2.1 Let $\Sigma_{i i}$, for $i=1, \ldots, p$, the marginal covariance functions of dimension $n \times n, \boldsymbol{\Sigma}_{b}$ a valid spatial correlation function of dimension $p \times p$ and $\mathbf{I}$ the identity matrix of dimension $n \times n$, then the covariance function defined in (2) is a valid and full rank $n p$ specification for multivariate spatial data modeling.

Proof Since the marginal covariance functions, $\Sigma_{i i}$, for $i=1, \ldots, p$, are symmetric positive definite matrix, the matrices $\tilde{\boldsymbol{\Sigma}}_{i i}$, resulting from the Cholesky decomposition, are lower triangular with positive diagonal elements and therefore, full rank (Banerjee and Roy, 2014). Let's denote by $R_{\tilde{\boldsymbol{\Sigma}}_{i i}}$, the rank of $\tilde{\boldsymbol{\Sigma}}_{i i}$. Thus, $\operatorname{rank}\left(\tilde{\boldsymbol{\Sigma}}_{i i}\right)=R_{\tilde{\boldsymbol{\Sigma}}_{i i}}=n$, for all $i$, and from the rank properties of the block-diagonal matrix, the rank of any block-diagonal matrix is the sum of the ranks of its diagonal blocks (Banerjee and Roy, 2014), that is:

$$
\operatorname{rank}\left[B_{\operatorname{diag}}\left(\tilde{\boldsymbol{\Sigma}}_{11}^{\top}, \tilde{\boldsymbol{\Sigma}}_{22}^{\top}, \ldots, \tilde{\boldsymbol{\Sigma}}_{p p}^{\top}\right)\right]=\sum_{i=1}^{p} R_{\tilde{\boldsymbol{\Sigma}}_{i i}}=n p,
$$

therefore, $B_{\operatorname{diag}}\left(\tilde{\boldsymbol{\Sigma}}_{11}^{\top}, \tilde{\boldsymbol{\Sigma}}_{22}^{\top}, \ldots, \tilde{\mathbf{\Sigma}}_{p p}^{\top}\right)$ is a full $n p$ rank matrix. 
On the other hand, since $\boldsymbol{\Sigma}_{b}$ and $\mathbf{I}$ are positive definite matrices, it follows by the kronecker product properties that $\left(\boldsymbol{\Sigma}_{b} \otimes \mathbf{I}\right)$ is also a positive definite matrix (Hardy and Steeb, 2019). With $\boldsymbol{\Sigma}_{b}$ of dimension $p$ and $\mathbf{I}$ of dimension $n$, the resulting kronecker product between them will be a positive definite matrix of dimension $n p$.

Now, for simplicity of notation, let's denote by $\mathbf{A}$ and $\mathbf{B}$ the respective $\left(\boldsymbol{\Sigma}_{b} \otimes \mathbf{I}\right)$ and $B_{\text {diag }}\left(\tilde{\boldsymbol{\Sigma}}_{11}^{\top}, \tilde{\boldsymbol{\Sigma}}_{22}^{\top}, \ldots, \tilde{\boldsymbol{\Sigma}}_{p p}^{\top}\right)$ matrices. Since $\mathbf{A}$ is a positive definite matrix and $\mathbf{B}$ is a full rank matrix, follows that $\mathbf{B}^{\top} \mathbf{A B}$ preserves not only the rank but also the positive definiteness (Gentle, 2017; Petersen et al, 2008). To visualize this, let $\mathbf{a} \neq \mathbf{0}$, any vector of dimension $n p$, and let $\mathbf{z}=\mathbf{B a}$, where $\mathbf{z} \neq \mathbf{0}$, because $\mathbf{B}$ is a full rank matrix. Using the positive definite matrix definition, follows:

$$
\begin{aligned}
\mathbf{a}^{\top}\left(\mathbf{B}^{\top} \mathbf{A B}\right) \mathbf{a} & =(\mathbf{a B})^{\top} \mathbf{A}(\mathbf{B a}) \\
& =\mathbf{z}^{\top} \mathbf{A} \mathbf{z} \\
& >0
\end{aligned}
$$

The result holds if variables are observed in different numbers of sample locations, since the incomplete data can be treated as missing information and this does not imply any additional complexity and the proof of theorem 2.1 remains valid.

The dimensions of the resulting covariance matrix will depend on the number of sample locations for each variable considered in the analysis. If we consider marginal covariance functions with dimension $n_{i}$, for $i=1, \ldots, p$, the resulting covariance matrix will have dimension $N=\sum_{i=1}^{p} n_{i}$.

Considering that $\boldsymbol{\Sigma}$ is a valid covariance specification for any valid choice of marginal-covariance and correlation functions, the proposed model allows its smoothing parameters, scale, correlation and variance to vary in their usual domains, favoring the inferential process and allowing the model parameters to be more easily interpreted.

To illustrate the behavior of the proposed model, we simulate a bivariate MatSimpler model on a regular unit grid. We set the parameter values as $\sigma_{1}=0.5, \sigma_{2}=1.0, \nu_{1}=0.5, \nu_{2}=0.8 \phi_{1}=0.1, \phi_{2}=0.2$ and $\rho_{12}=-0.7$ or 0.7 , aiming to illustrate different smoothness and variability in the data and situations with positive and negative correlations between the variables. Fig. 1 shows the corresponding simulations with the corresponding code available in the on-line supplementary material.

\subsection{Estimation and inference}

For the estimation process, let $N=n p$ and $\mathbf{Y}=\left\{\mathbf{Y}_{1}^{\top}, \ldots, \mathbf{Y}_{p}^{\top}\right\}^{\top}$, be the $N \times 1$ stacked vector of response variables, with $N \times 1$ mean-vector $\boldsymbol{\mu}=$ $\left\{\boldsymbol{\mu}_{1}^{\top}, \ldots, \boldsymbol{\mu}_{p}^{\top}\right\}^{\top}$, where $\boldsymbol{\mu}_{i}=\mathbf{X}_{i} \boldsymbol{\beta}_{i}$ denotes the $n \times 1$ vector of expected values for the response variable $\mathbf{Y}_{i}, i=1, \ldots, p$, with $\mathbf{X}_{i}$ being a $n \times k_{i}$ design matrix composed of $k_{i}$ covariates and, consequently, $\boldsymbol{\beta}_{i}$ denotes a $k_{i} \times 1$ regression parameter vector. 

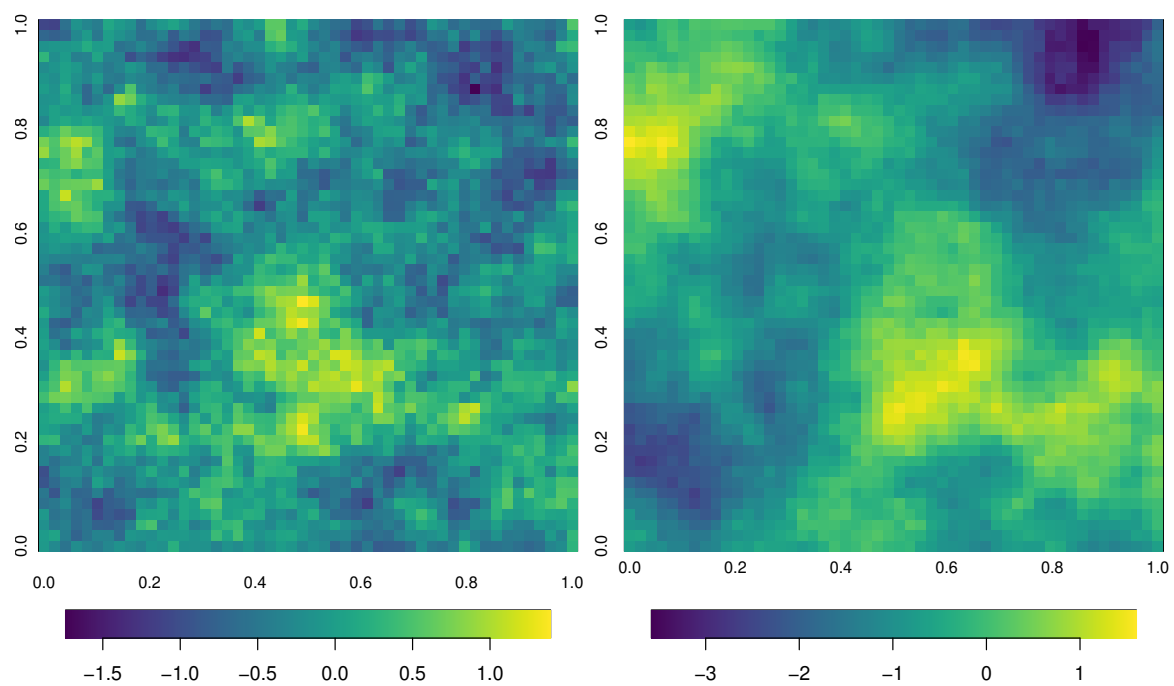

(A)
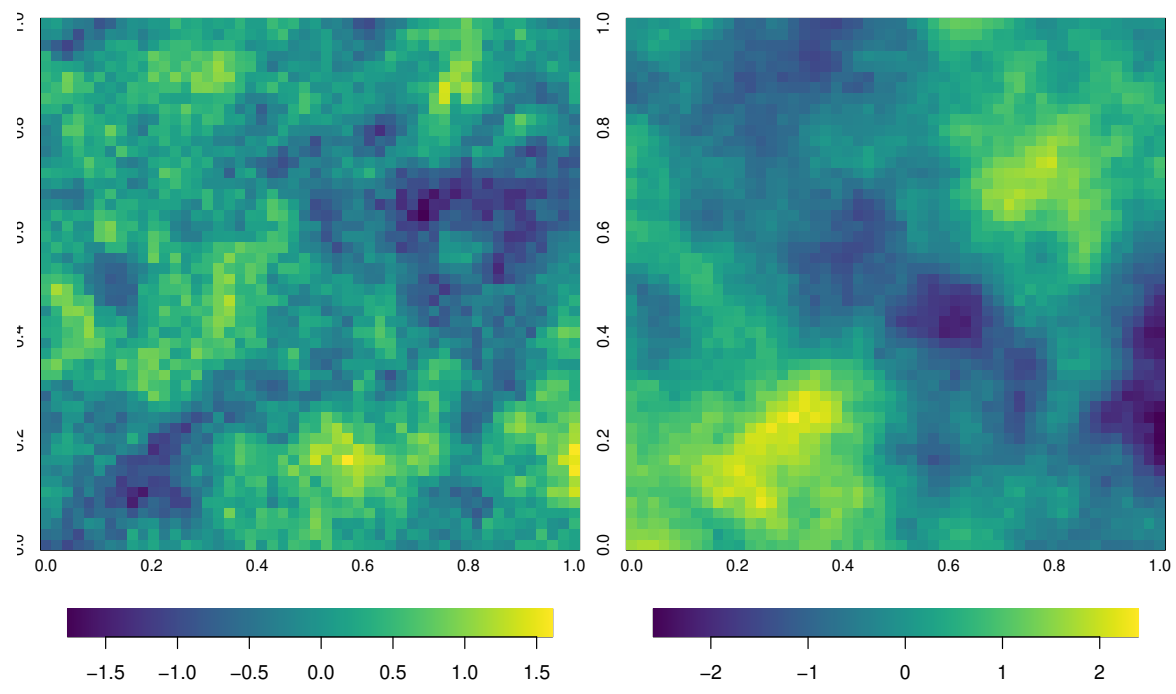

(B)

Fig. 1 Simulation of a bivariate MatSimpler model with (A) $\sigma_{1}=0.5, \sigma_{2}=$ $1.0, \nu_{1}=0.5, \nu_{2}=0.8 \phi_{1}=0.1, \phi_{2}=0.2$ and $\rho_{12}=0.7$ and (B) $\sigma_{1}=0.5$, $\sigma_{2}=1.0, \nu_{1}=0.5, \nu_{2}=0.8 \phi_{1}=0.1, \phi_{2}=0.2$ and $\rho_{12}=-0.7$

We will denote the set of parameters to be estimated by $\boldsymbol{\theta}=\left(\boldsymbol{\beta}^{\top}, \boldsymbol{\lambda}^{\top}\right)^{\top}$, where $\boldsymbol{\beta}=\left(\boldsymbol{\beta}_{1}^{\top}, \ldots, \boldsymbol{\beta}_{p}^{\top}\right)^{\top}$ denotes the regression parameters vector and $\boldsymbol{\lambda}=$ 
$\left(\rho_{1}, \ldots, \rho_{p(p-1) / 2}, \sigma_{1}, \ldots, \sigma_{p}, \nu_{1}, \ldots, \nu_{p}, \phi_{1}, \ldots, \phi_{p}\right)^{\top}$ is the covariance specification parameters vector. Considering $\mathbf{y}$ the stacked vector of observed values, the $\log$-likelihood function for $\boldsymbol{\theta}$ is given by:

$$
\mathcal{L}(\boldsymbol{\theta} ; \mathbf{y})=-\frac{1}{2}\left[N \ln (2 \pi)+\ln |\boldsymbol{\Sigma}(\boldsymbol{\lambda})|+(\mathbf{y}-\boldsymbol{\mu}(\boldsymbol{\beta}))^{\top} \boldsymbol{\Sigma}(\boldsymbol{\lambda})^{-1}(\mathbf{y}-\boldsymbol{\mu}(\boldsymbol{\beta}))\right] .
$$

The vector of expected values, $\boldsymbol{\mu}(\boldsymbol{\beta})$, depends on the regression parameters while the covariance specification $\boldsymbol{\Sigma}(\boldsymbol{\lambda})$ depends on the covariance parameters vector, $\boldsymbol{\lambda}$. We obtain the maximum likelihood estimators by maximizing the function in (5) with respect to the $\boldsymbol{\theta}$ parameter vector.

Based on matrix properties (Wand, 2002; Bonat et al, 2020), the score functions with respect to the $\beta$ and $\boldsymbol{\lambda}$ parameters, are given, respectively, by:

$$
\begin{gathered}
\mathcal{U}_{\boldsymbol{\beta}}=\mathbf{D}^{\top} \boldsymbol{\Sigma}(\boldsymbol{\lambda})^{-1} \mathbf{r}(\boldsymbol{\beta}), \\
\mathcal{U}_{\boldsymbol{\lambda}}=-\frac{1}{2}\left\{\boldsymbol{\Sigma}(\boldsymbol{\lambda})^{-1}-\boldsymbol{\Sigma}(\boldsymbol{\lambda})^{-1} \mathbf{r}(\boldsymbol{\beta}) \mathbf{r}(\boldsymbol{\beta})^{\top} \boldsymbol{\Sigma}(\boldsymbol{\lambda})^{-1}\right\} \frac{\partial \boldsymbol{\Sigma}(\boldsymbol{\lambda})}{\partial \boldsymbol{\lambda}},
\end{gathered}
$$

with $\mathbf{r}(\boldsymbol{\beta})=(\mathbf{y}-\boldsymbol{\mu}(\boldsymbol{\beta}))$ and $\mathbf{D}=\frac{\partial \boldsymbol{\mu}(\boldsymbol{\beta})}{\partial \boldsymbol{\beta}}=\operatorname{Bdiag}\left(\mathbf{X}_{1}, \ldots, \mathbf{X}_{p}\right)$.

We achieve the maximum likelihood estimator of $\beta$ by solving $\mathcal{U}_{\beta}$, which results in:

$$
\hat{\boldsymbol{\beta}}=\left(\mathbf{D}^{\top} \boldsymbol{\Sigma}(\boldsymbol{\lambda})^{-1} \mathbf{D}\right)^{-1}\left(\mathbf{D}^{\top} \boldsymbol{\Sigma}(\boldsymbol{\lambda})^{-1} \mathbf{y}\right) .
$$

Making similar calculations, we find the Fisher information matrix which, for $\beta$, is given by:

$$
\mathcal{F}_{\boldsymbol{\beta}}=\mathbf{D}^{\top} \boldsymbol{\Sigma}(\boldsymbol{\lambda})^{-1} \mathbf{D} .
$$

For $\boldsymbol{\lambda}$, the $(i, j)^{t h}$ entry of the Fisher information matrix, is given by:

$$
\left[\mathcal{F}_{\boldsymbol{\lambda}}\right]_{i j}=\frac{1}{2} \operatorname{tr}\left[\mathbf{W}_{\lambda_{i}} \boldsymbol{\Sigma}(\boldsymbol{\lambda}) \mathbf{W}_{\lambda_{j}} \boldsymbol{\Sigma}(\boldsymbol{\lambda})\right],
$$

where $\mathbf{W}_{\lambda_{i}}=-\partial \boldsymbol{\Sigma}(\boldsymbol{\lambda})^{-1} / \partial \lambda_{i}$.

Considering $\hat{\boldsymbol{\theta}}=\left(\hat{\boldsymbol{\beta}}^{\top}, \hat{\boldsymbol{\lambda}}^{\top}\right)^{\top}$ the maximum likelihood estimator of $\boldsymbol{\theta}$ parameter, we have that its asymptotic distribution is $\hat{\boldsymbol{\theta}} \sim N\left(\boldsymbol{\theta}, \mathcal{F}_{\theta}^{-1}\right)$, where $\mathcal{F}_{\boldsymbol{\theta}}=\left(\begin{array}{cc}\mathcal{F}_{\boldsymbol{\beta}} & \mathbf{0} \\ \mathbf{0} & \mathcal{F}_{\boldsymbol{\lambda}}\end{array}\right)$ denotes the Fisher information matrix of $\boldsymbol{\theta}$.

The maximum likelihood estimates of $\boldsymbol{\lambda}$ can be found through Newton's scoring iterative algorithm (Bonat et al, 2020):

$$
\lambda^{i+1}=\lambda^{i}-\alpha \mathcal{F}_{\boldsymbol{\lambda}}^{-1} \mathcal{U}_{\boldsymbol{\lambda}}(\tilde{\boldsymbol{\theta}} ; \mathbf{y}),
$$

where $\tilde{\boldsymbol{\theta}}=\left(\hat{\boldsymbol{\beta}}^{\top}, \boldsymbol{\lambda}^{\top}\right)^{\top}$ and $\alpha$ controls the step length.

In Section 4 through a simulation study we evaluate the asymptotic properties of the maximum likelihood estimators for the model in a finite sample scenario. 


\subsection{Derivatives of the covariance matrix}

We present in this subsection the derivative of matrix $\boldsymbol{\Sigma}$ used in calculations of (6). Let $\rho_{r}$, for $r=1, \ldots, p(p-1) / 2$, denoting the correlation parameters of $\boldsymbol{\Sigma}_{b}, \sigma_{i}^{2}, \phi_{i}$ and $\nu_{i}$, denoting the variance, scale and smoothness parameters of the marginal-covariance matrix, $\Sigma_{i i}$, for $i=1, \ldots, p$.

The partial derivative of the matrix-valued covariance function $\boldsymbol{\Sigma}$, with respect to each correlation parameter, $\rho_{r}$, is given by:

$$
\frac{\partial \boldsymbol{\Sigma}}{\partial \rho_{r}}=B_{\operatorname{diag}}\left(\tilde{\boldsymbol{\Sigma}}_{11}, \tilde{\boldsymbol{\Sigma}}_{22}, \ldots, \tilde{\boldsymbol{\Sigma}}_{p p}\right)\left(\frac{\partial \boldsymbol{\Sigma}_{b}}{\partial \rho_{r}} \otimes \mathbf{I}\right) B_{\operatorname{diag}}\left(\tilde{\boldsymbol{\Sigma}}_{11}^{\top}, \tilde{\boldsymbol{\Sigma}}_{22}^{\top}, \ldots, \tilde{\boldsymbol{\Sigma}}_{p p}^{\top}\right)
$$

To obtain the partial derivative with respect to variance parameter, $\sigma_{i}^{2}$, we will use matrix properties, that is,

$$
\begin{aligned}
\frac{\partial \boldsymbol{\Sigma}}{\partial \sigma_{i}^{2}} & =B_{\operatorname{diag}}\left(\mathbf{0}, \ldots, \frac{\partial \tilde{\boldsymbol{\Sigma}}_{i i}}{\partial \sigma_{i}^{2}}, \ldots, \mathbf{0}\right)\left(\boldsymbol{\Sigma}_{b} \otimes \mathbf{I}\right) B_{\operatorname{diag}}\left(\tilde{\boldsymbol{\Sigma}}_{11}^{\top}, \tilde{\boldsymbol{\Sigma}}_{22}^{\top}, \ldots, \tilde{\boldsymbol{\Sigma}}_{p p}^{\top}\right) \\
& +B_{\operatorname{diag}}\left(\tilde{\boldsymbol{\Sigma}}_{11}, \tilde{\boldsymbol{\Sigma}}_{22}, \ldots, \tilde{\boldsymbol{\Sigma}}_{p p}\right)\left(\boldsymbol{\Sigma}_{b} \otimes \mathbf{I}\right) B_{\operatorname{diag}}\left(\mathbf{0}, \ldots, \frac{\partial \tilde{\boldsymbol{\Sigma}}_{i i}^{\top}}{\partial \sigma_{i}^{2}}, \ldots, \mathbf{0}\right)
\end{aligned}
$$

An analogous procedure to the Eq. (7) can be used to obtain the derivatives with respect to $\phi_{i}$ and $\nu_{i}$. Thus, to obtain the derivatives of $\boldsymbol{\Sigma}$ with respect to each parameter, we must calculate the parcial derivatives in (7). Using the result of partial derivatives of Cholesky's factorization (Särkkä, 2013; Bonat and Jørgensen, 2016), follows:

$$
\begin{aligned}
\frac{\partial \tilde{\boldsymbol{\Sigma}}_{i i}}{\partial \sigma_{i}^{2}} & =\tilde{\boldsymbol{\Sigma}}_{i i} \Phi\left(\tilde{\boldsymbol{\Sigma}}_{i i}^{-1} \frac{\partial \Sigma_{i i}}{\partial \sigma_{i}^{2}} \tilde{\boldsymbol{\Sigma}}_{i i}^{-1}\right), \\
\frac{\partial \tilde{\boldsymbol{\Sigma}}_{i i}}{\partial \phi_{i}} & =\tilde{\boldsymbol{\Sigma}}_{i i} \Phi\left(\tilde{\boldsymbol{\Sigma}}_{i i}^{-1} \frac{\partial \Sigma_{i i}}{\partial \phi_{i}} \tilde{\boldsymbol{\Sigma}}_{i i}^{-1}\right), \\
\frac{\partial \tilde{\boldsymbol{\Sigma}}_{i i}}{\partial \nu_{i}} & =\tilde{\boldsymbol{\Sigma}}_{i i} \Phi\left(\tilde{\boldsymbol{\Sigma}}_{i i}^{-1} \frac{\partial \Sigma_{i i}}{\partial \nu_{i}} \tilde{\boldsymbol{\Sigma}}_{i i}^{-1}\right),
\end{aligned}
$$

where $\Phi($.$) is the strictly lower triangular part of the argument and half of its$ diagonal. 


\subsection{Computational results}

As already mentioned, the MatSimpler model can be extended for more than two variables with relative ease. The computational time estimation will depend on the number of variables and sample locations considered in the analysis. To illustrate the computational time spent on estimation, we implement the generic model for $p$ variables and $n$ sample locations and simulate scenarios of the MatSimpler model considering different sample sizes and variable numbers. To make the simulation process easier, we set $\phi_{i}=0.2, \nu_{i}=0.5$, $\sigma_{i}=0.3$, for all $i=1, \ldots, p$. The correlation parameters were chosen between -0.7 to 0.7 such that the resulting $\boldsymbol{\Sigma}_{b}$ was a valid structure. We consider the number of variables, $p$, ranging from 2 to 6 and the number of sample locations, $n=(100,225,400,625,900)$, taken in a unit square grid.

The results, presented in Appendix A (Fig. 8) shows that the estimation computational time increases with the number of variables and sample locations, which is due to the Cholesky decompositions of the marginal-covariance matrices. These calculations are necessary to obtain the proposed specification.

We also compare the estimation computational times for the bivariate case of the MatSimpler model with three other literature models, the $b i$ variate Matérn model with constraints (MatConstr), the bivariate separable Matérn model (MatSep) and the LMC model. The data were simulated from the MatConstr model. We set $\phi_{i}=0.2, \nu_{i}=0.5, \sigma_{i}=0.3$, for $i=1,2$, and $\rho_{12}=0.8$. The MatConstr, MatSep and LMC models were estimated by GeoModels package, in which we consider the standard likelihood function. For all models we used the Nelder-Mead optimizer and convergence was successful in all scenarios. Fig. 9 in Appendix A illustrates the comparisons between models.

Based on the simulations carried out, we see that the MatSimpler model, with 7 parameters, presents estimation times much lower than the MatConstr model which have the same number of parameters. Furthermore, our model presents estimation times very close to the MatSep model (with 5 parameters), especially for sample locations less than 400, and to the LMC (with 6 parameters). The $\mathrm{R}$ codes and the simulation results presented in Figures 8 and 9 are available in the supplementary material.

\subsection{Prediction}

For the case of multivariate spatial data, spatial prediction is a generalization of the univariate case that consists of predicting $\mathbf{Y}$ at some unknown location, $\mathbf{s}_{0}$, based on other sample information, $\mathbf{s}_{i}$, for $i=1, \ldots, n$ (Ver Hoef and Cressie, 1993; Bivand et al, 2008; Pebesma, 2004).

Let $\boldsymbol{\Sigma}_{\mathbf{Y}_{1}}$ be the covariance matrix of $\mathbf{Y}_{1}=\mathbf{Y}\left(\mathbf{s}_{i}\right)$, for $i=1, \ldots, n, \boldsymbol{\Sigma}_{\mathbf{Y}_{0}}$ be the covariance matrix of $\mathbf{Y}_{0}=\mathbf{Y}\left(\mathbf{s}_{0}\right), \boldsymbol{\Sigma}_{\mathbf{Y}_{1} \mathbf{Y}_{0}}$ be the covariance matrix between $\mathbf{Y}_{1}$ and $\mathbf{Y}_{0}$ and $\mathbf{d}_{0}=\operatorname{Bdiag}\left(\mathbf{x}_{1}\left(\mathbf{s}_{0}\right), \ldots, \mathbf{x}_{p}\left(\mathbf{s}_{0}\right)\right)$. Then, the best linear unbiased predictor for $\mathbf{Y}_{0}$ is: 


$$
E\left(\mathbf{Y}_{0} \mid \mathbf{Y}_{1}\right)=E\left(\mathbf{Y}_{0}\right)+\boldsymbol{\Sigma}_{\mathbf{Y}_{1} \mathbf{Y}_{0}}^{\top} \boldsymbol{\Sigma}_{\mathbf{Y}_{1}}^{-1}\left(\mathbf{Y}_{1}-E\left(\mathbf{Y}_{1}\right)\right)
$$

with prediction covariance matrix:

$$
\operatorname{Cov}\left(\mathbf{Y}_{0} \mid \mathbf{Y}_{1}\right)=\boldsymbol{\Sigma}_{\mathbf{Y}_{0}}-\boldsymbol{\Sigma}_{\mathbf{Y}_{1} \mathbf{Y}_{0}}^{\top} \boldsymbol{\Sigma}_{\mathbf{Y}_{1}}^{-1} \boldsymbol{\Sigma}_{\mathbf{Y}_{1} \mathbf{Y}_{0}}
$$

We can replace the unknown parameters of the model by their respective maximum likelihood estimators (Martins et al, 2016) and, with this, we obtain the stacked vector prediction for the $p$ variables in the $\mathbf{s}_{0}$ unobserved locations.

\subsection{Computational resources}

In our computational implementation, we used the $\mathrm{R}$ statistical software $(\mathrm{R}$ Core Team, 2021) for the estimation, simulation and prediction procedures. For the implementation of the proposed covariance matrix specification, we used the kronecker function, basic to R, which enables the calculation of the kronecker product between the matrices $\boldsymbol{\Sigma}_{b}$ and $\mathbf{I}$. We use the Matrix (Bates and Maechler, 2021) package to perform matrix operations more efficiently, such as the Cholesky decomposition of the marginal-covariance matrices, through the chol function, and the construction of the diagonal block matrix $B_{\text {diag }}\left(\tilde{\boldsymbol{\Sigma}}_{11}, \tilde{\boldsymbol{\Sigma}}_{22}, \ldots, \tilde{\boldsymbol{\Sigma}}_{p p}\right)$, through the bdiag function.

To calculate the marginal-covariance functions, we use the matern function from geoR (Ribeiro Jr et al, 2020) package. Furthermore, the crossprod and tcrossprod functions allowed a more efficient calculation of products between matrices. To calculate the log-likelihood function, used in the estimation process, we use the dmvn function and for simulations we use the rmvn function, both from mvnfast package (Fasiolo, 2016) that provides computationally efficient methods related to the multivariate normal and Student's t distributions. For the log-likelihood function optimization, we use optim function. R codes are available in on-line supplementary material.

\section{Data analysis}

This section illustrates the application of the proposed model in two datasets. For the first one, the soill dataset, aim is to model the hydrogen content and the catium exchange capability (CTC). We compare results obtained fitting the MatSimpler and other models. The second one, a dataset on heavy metal concentration, is used to illustrate the model's flexibility by fitting a fourvariate dataset.

\subsection{Example 1: Soil data}

The soil250 dataset from geoR package (Ribeiro Jr et al, 2020) contains some soil chemistry properties measured on a regular grid with $10 \mathrm{x} 25$ points spaced by 5 meters. We study the relation between the hydrogen content and the catium exchange capability (CTC). These data illustrate a scenario with strong 
correlation between the variables under study. Fig. 2 shows circle plots of the hydrogen (left panel) and CTC (right panel) data separately. The coordinates are divided by a constant to easy the visualizations.

Fig. 3 shows the histograms of each variable. Scatterplots of each variable against each spatial coordinate in Fig. 4 are used to check for spatial trends. We proceed with the residuals of a linear regression with constant trend as a realization of a zero-mean bivariate isotropic stationary Gaussian random field. The sample correlation between the variables was 0.68. Standard deviations are 0.60 for hydrogen content and 0.77 for the CTC.

The models considered in the estimation step are the MatSimpler, LMC, MatConstr, MatInd and MatSep. For MatSimpler and MatConstr the vector of parameters to be estimated is $\boldsymbol{\lambda}=\left(\phi_{1}, \phi_{2}, \nu_{1}, \nu_{2}, \sigma_{1}, \sigma_{2}, \rho_{12}\right)$, for the separable model, MatSep, we have $\phi_{1}=\phi_{2}$ and $\nu_{1}=\nu_{2}$ and for the independent model we have $\rho_{12}=0$. The LMC model is parameterized by $\boldsymbol{\lambda}=\left(a_{11}, a_{12}, a_{22}, a_{21}, \phi_{1}, \phi_{2}\right)$, where $a_{11}, a_{12}, a_{22}$ and $a_{21}$ are elements of the corregionalization matrix and $\phi_{1}$ and $\phi_{2}$ are parameters of the exponential correlation model. Table 1 presents the parameter estimates, the maximized log-likelihood (LL), the Akaike information criterion (AIC) and the Bayesian information criterion (BIC) values considering each model. The MatConstr, MatInd, LMC and MatSep models are estimated using the GeoFit function from the GeoModels package (Bevilacqua and Morales-Oñate, 2018) for which the standard likelihood function and the Euclidean distance were considered. The Nelder-Mead optimizer is the algorithm of choice for all model fits. Table 2 presents the estimation times. The $\mathrm{R}$ codes for the Tables 1-2 are available in the supplementary material.
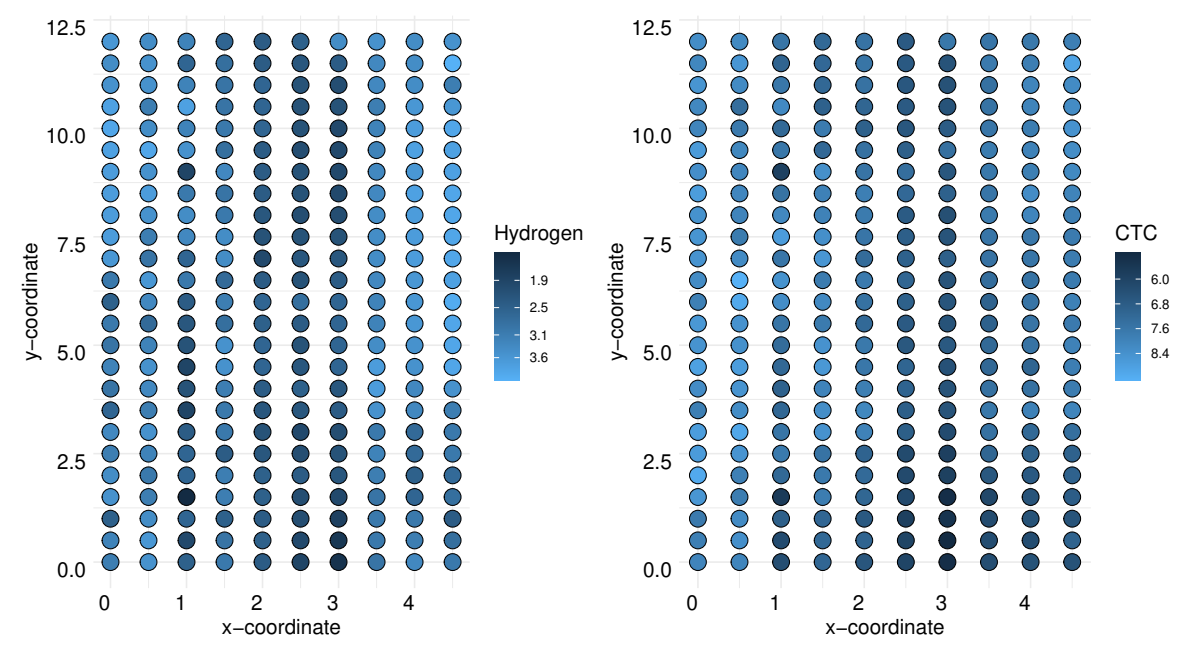

Fig. 2 Circle plot of the (A) Hydrogen content and (B) CTC for soil250 data 
(A)

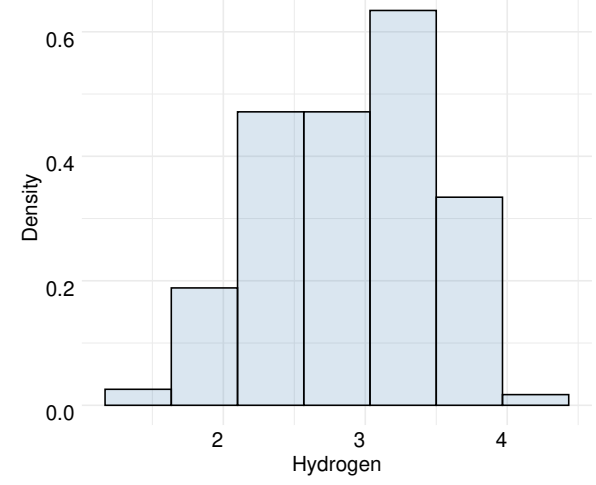

(B)

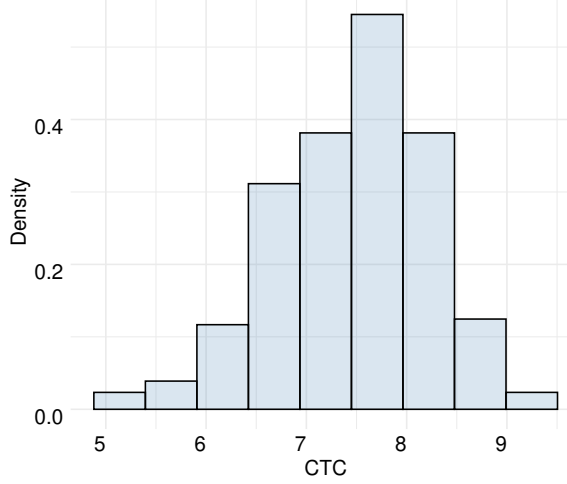

Fig. 3 Histogram of (A) Hydrogen content and (B) CTC for soil250 data

(A)

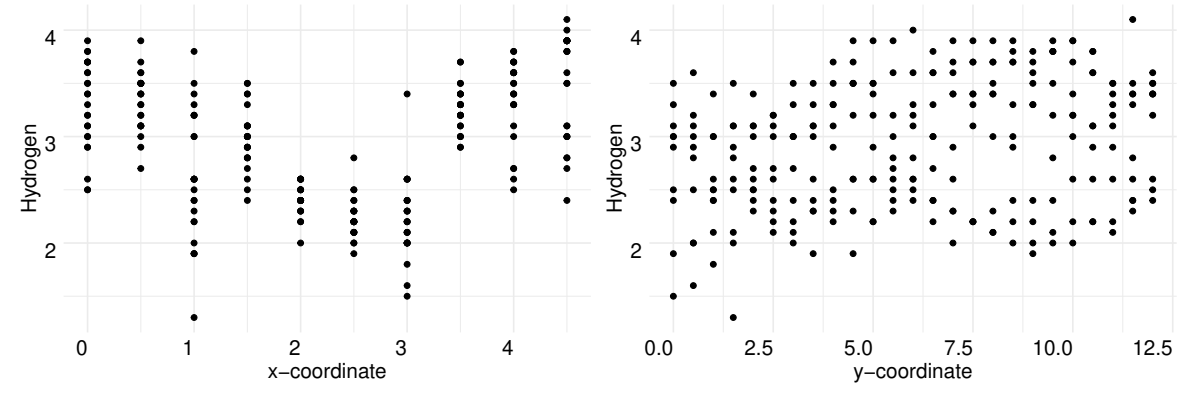

(B)

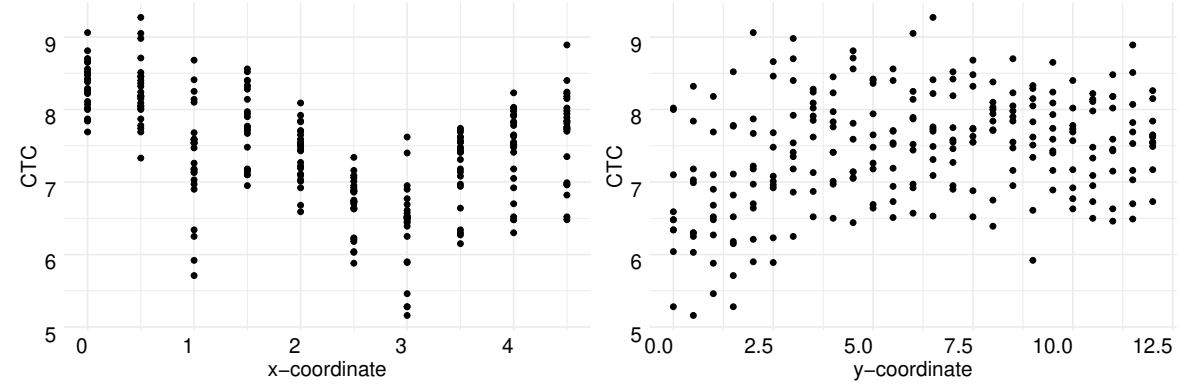

Fig. 4 Scatterplots of (A) Hydrogen content and (B) CTC against the coordinates for soil250 data

The predictive behavior was assessed by a random training selection of 200 locations ( $80 \%$ of the data), from which we estimate the models under study and compute the mean absolute error (MAE), the root mean square error (RMSE) and the normalized mean square error (NMSE) for each model using cokriging predictor for the 50 remaining locations (20\% of the data). These 
Table 1 Parameter estimates of each model for soil250 data

\begin{tabular}{cccccc}
\hline & \multicolumn{5}{c}{ Models } \\
\cline { 2 - 6 } Estimates & MatSimpler & MatConstr & MatInd & LMC & MatSep \\
\hline$a_{11}$ & - & - & - & 0.615 & - \\
$a_{12}$ & - & - & - & -0.067 & - \\
$a_{22}$ & - & - & - & 0.544 & - \\
$a_{21}$ & - & 1.415 & 1.849 & 0.608 & - \\
$\hat{\phi}_{1}$ & 1.077 & 1.483 & 2.340 & 2.816 & 1.978 \\
$\hat{\phi}_{2}$ & 1.994 & 0.509 & 0.398 & - & \\
$\nu_{1}$ & 0.543 & 0.562 & 0.422 & - & 0.495 \\
$\nu_{2}$ & 0.513 & 0.671 & 0.648 & - & 0.774 \\
$\hat{\sigma_{1}}$ & 0.627 & 0.850 & 0.848 & - & 0.891 \\
$\hat{\sigma_{2}}$ & 0.920 & 0.811 & - & - & 0.816 \\
$\hat{\rho}_{12}$ & 0.823 & -166.143 & -301.813 & -164.688 & -166.298 \\
\hline LL & -161.551 & 346.285 & 615.626 & 341.376 & 342.595 \\
AIC & 337.103 & 375.787 & 640.914 & 366.663 & 363.668 \\
BIC & 361.753 & & & & \\
\hline
\end{tabular}

Table 2 Estimation times for each model for soil250 data

\begin{tabular}{cccccc}
\hline & \multicolumn{5}{c}{ Models } \\
\cline { 2 - 6 } Estimates & MatSimpler & MatConstr & MatInd & LMC & MatSep \\
\hline User & 109.918 & 405.609 & 169.595 & 96.770 & 113.649 \\
System & 0.442 & 0.588 & 0.120 & 0.253 & 0.124 \\
Elapsed & 59.301 & 406.421 & 169.630 & 97.002 & 113.752 \\
\hline N. Param & 7 & 7 & 6 & 6 & 5 \\
\hline
\end{tabular}

measures are defined as,

$$
\begin{aligned}
\operatorname{MAE}_{i} & =\frac{1}{50} \sum_{k=1}^{50}\left|X_{i}\left(\mathbf{s}_{k}\right)-\hat{X}_{i}\left(\mathbf{s}_{k}\right)\right|, \\
\operatorname{RMSE}_{i} & =\sqrt{\frac{1}{50} \sum_{k=1}^{50}\left(X_{i}\left(\mathbf{s}_{k}\right)-\hat{X}_{i}\left(\mathbf{s}_{k}\right)\right)^{2},} \\
\mathrm{NMSE}_{i} & =\frac{\mathrm{RMSE}_{i}}{\max \left(\hat{X}_{i}\left(\mathbf{s}_{k}\right)\right)-\min \left(\hat{X}_{i}\left(\mathbf{s}_{k}\right)\right)},
\end{aligned}
$$

where $\hat{X}_{i}\left(\mathbf{s}_{k}\right)$ is the cokriging predictor of the variable $X_{i}\left(\mathbf{s}_{k}\right)$, with $i=$ $\mathrm{H}, \mathrm{CTC}$, representing hydrogen content and CTC, respectively. We repeated the same process 150 times, calculating the values of the $\mathrm{MAE}_{i}, \mathrm{RMSE}_{i}$ and $\mathrm{NMSE}_{i}$, for each variable each time. Table 3 presents the mean and standard 
deviation for the prediction errors considering each model estimated for each variable.

The MatSimpler model showed a better fit when compared to the other models with respect to log-likelihood, AIC and BIC values. The estimation times of the MatSimpler model were also lower, especially when compared to the MatConstr model which has the same number of parameters. Thus, we observe that the proposed MatSimpler model is a competitive model with the classical models in the literature.

Table 3 Mean and standard deviation (sd) for prediction errors considering 150 splits of data into training (80\%) and test (20\%) for each model and each variable for soil250 data

\begin{tabular}{cccccccc}
\hline Models & & MAE $_{\mathrm{H}}$ & $\mathrm{RMSE}_{\mathrm{H}}$ & $\mathrm{NMSE}_{\mathrm{H}}$ & $\mathrm{MAE}_{\mathrm{CTC}}$ & $\mathrm{RMSE}_{\mathrm{CTC}}$ & NMSE $_{\mathrm{CTC}}$ \\
\hline \multirow{2}{*}{ MatSimpler } & Mean & 0.277 & 0.367 & 0.215 & 0.314 & 0.416 & 0.151 \\
& sd & 0.032 & 0.045 & 0.032 & 0.032 & 0.049 & 0.023 \\
MatConstr & Mean & 0.271 & 0.364 & 0.204 & 0.307 & 0.410 & 0.150 \\
& sd & 0.032 & 0.046 & 0.030 & 0.034 & 0.052 & 0.024 \\
\multirow{2}{*}{ MatInd } & Mean & 0.271 & 0.363 & 0.210 & 0.308 & 0.411 & 0.156 \\
& sd & 0.032 & 0.045 & 0.029 & 0.034 & 0.051 & 0.024 \\
LMC & Mean & 0.271 & 0.363 & 0.208 & 0.307 & 0.409 & 0.153 \\
& sd & 0.032 & 0.046 & 0.030 & 0.034 & 0.051 & 0.025 \\
MatSep & Mean & 0.270 & 0.363 & 0.202 & 0.307 & 0.409 & 0.152 \\
& sd & 0.033 & 0.047 & 0.030 & 0.034 & 0.052 & 0.024 \\
\hline
\end{tabular}

\subsection{Example 2: Heavy metal concentration data}

To illustrate the application of the MatSimpler model to a 4-variate set, we consider the data meuse dataset from the sp package, which contains topsoil heavy metal concentrations, collected in a plain flood of the river Meuse an area of approximately $15 \mathrm{~m} \times 15 \mathrm{~m}$. We studied the relationship between the variables cadmium, copper, lead, zinc, which have a strong sample correlation with each other. Fig. 5 shows the concentrations of each metal along the region under study.

One of the important predictors of heavy metal concentrations is the distance to the Meuse river. Thus, we consider such trend we take residuals from a linear fit of the metal concentrations on such distance. Considering $\hat{\rho}_{i j}$ the sample correlation between the variables $i$ and $j$, for $i, j=1, \ldots, 4$, the correlations between them are: $\hat{\rho}_{12}=0.6510, \hat{\rho}_{13}=0.6165, \hat{\rho}_{14}=0.6585, \hat{\rho}_{23}=$ 0.6970, $\hat{\rho}_{24}=0.7466, \hat{\rho}_{34}=0.9392$, with standard deviations: $\hat{s}_{1}=0.8627$, $\hat{s}_{2}=0.3343, \hat{s}_{3}=0.4625$ and $\hat{s}_{4}=0.4339$. The histograms of each quantity are shown in Fig. 6.

The parametric estimates as well as the standard errors for the MatSimpler model are presented in Table 4. The model was able to capture the individual variability of each variable as well as the estimated correlations between them. 

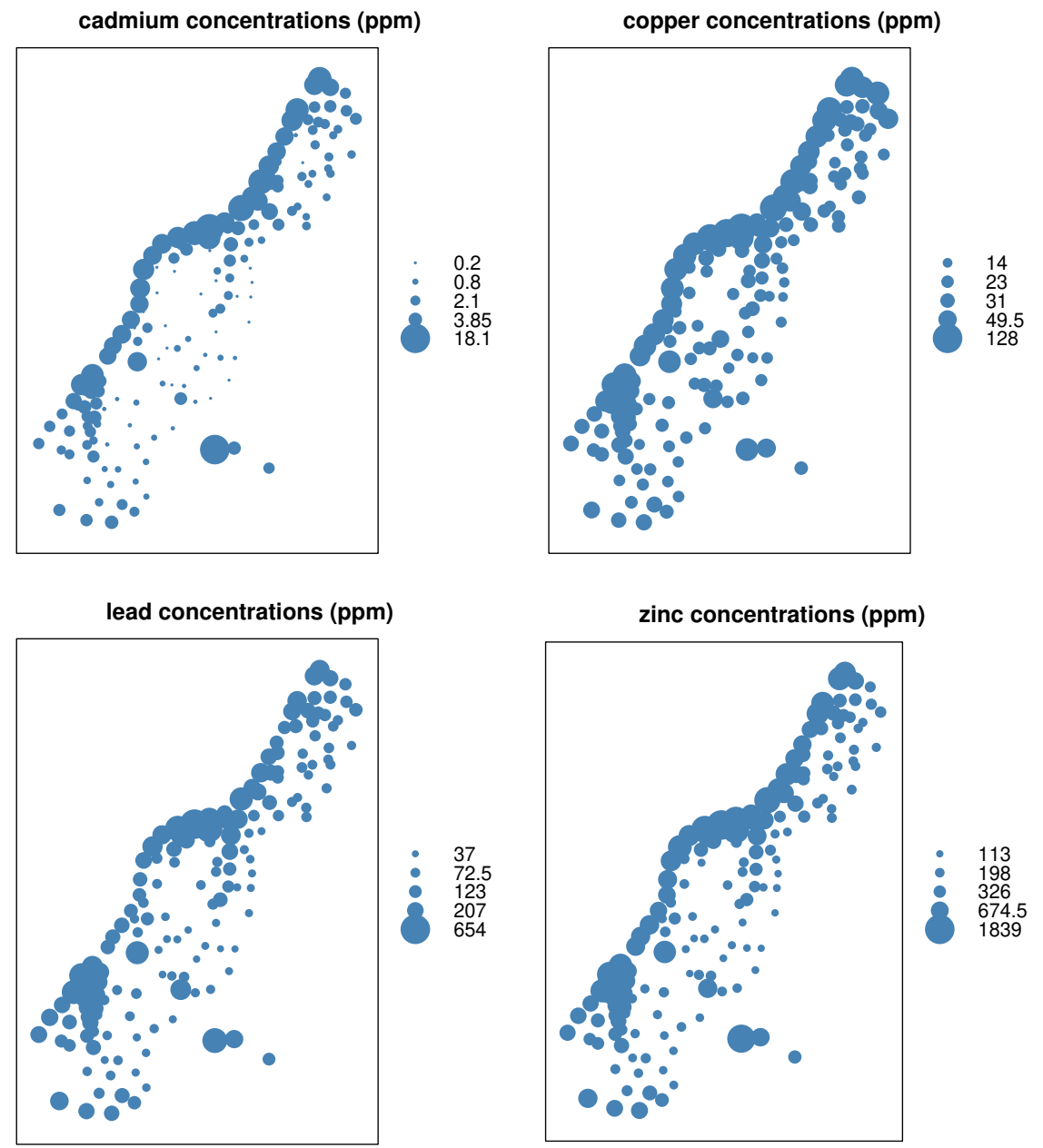

Fig. 5 Circle plot of (A) cadmium, (B) copper, (C) lead and (D) zinc concentration for meuse data

\section{Simulation study}

This section presents a simulation study to evaluate the behavior of the maximum likelihood estimators for the model parameters of the MatSimpler model. We explored a bivariate random field in three different scenarios to illustrate different situations that could occur in practice, exemplifying the flexibility of the proposed model in each case. Table 5 summarizes the simulated scenarios.

Scenario 1 considers the variables have smaller variability, for which we consider relatively small values for the variance parameters: $\sigma_{1}^{2}=0.25$ and 
(A)

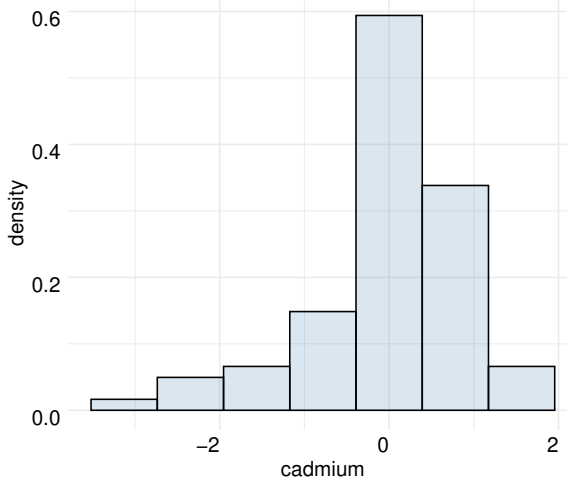

(C)

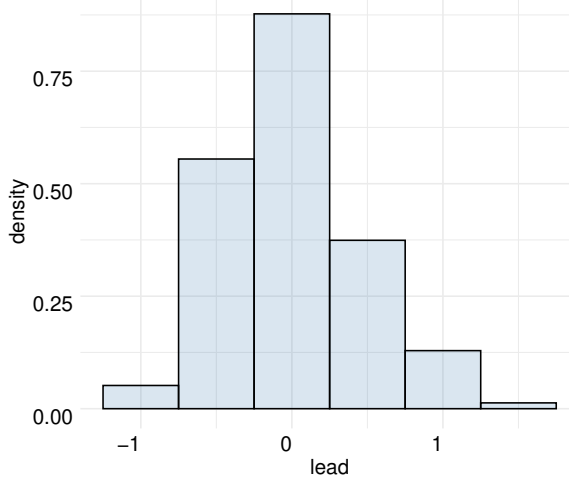

(B)

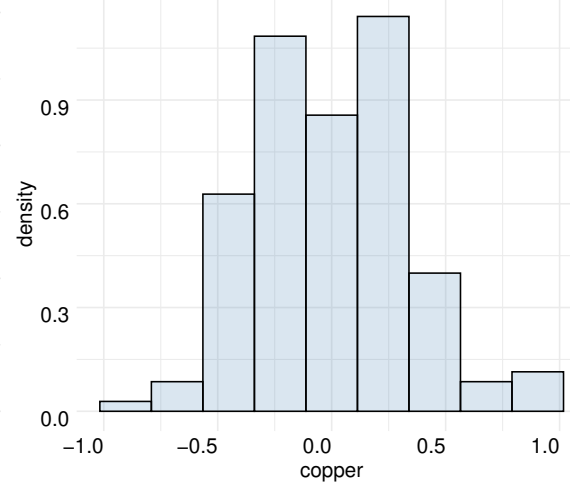

(D)

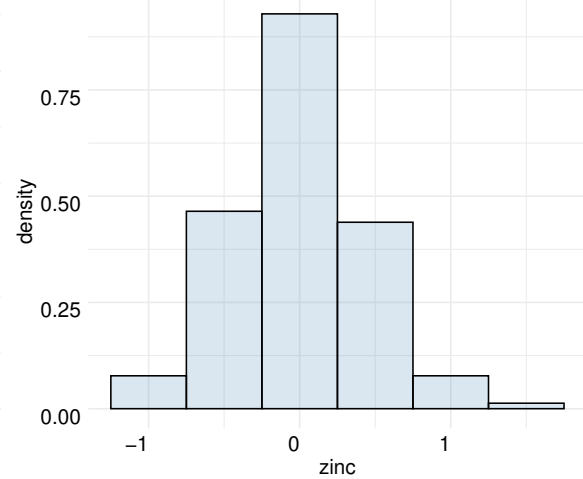

Fig. 6 Histogram of the residuals after removing tendencies with respect to distance of (A) cadmium, (B) copper, (C) lead and (D) zinc concentration

Table 4 Parameter estimates for meuse dataset

\begin{tabular}{ccccccc}
\hline & \multicolumn{6}{c}{ Estimates } \\
\cline { 2 - 7 } & $\hat{\phi}_{1}$ & $\hat{\phi}_{2}$ & $\hat{\phi}_{3}$ & $\hat{\phi}_{4}$ & $\hat{\nu}_{1}$ & $\hat{\nu}_{2}$ \\
\hline Value & 0.3953 & 0.8337 & 0.3959 & 0.3948 & 0.0699 & 0.0917 \\
sd & 0.2351 & 0.4761 & 0.1529 & 0.1663 & 0.0386 & 0.0417 \\
\hline & $\hat{\nu}_{3}$ & $\hat{\nu}_{4}$ & $\hat{s}_{1}$ & $\hat{s}_{2}$ & $\hat{s}_{3}$ & $\hat{s}_{4}$ \\
\cline { 2 - 7 } Value & 0.1407 & 0.1486 & 0.8650 & 0.3590 & 0.4369 & 0.4250 \\
sd & 0.0452 & 0.0508 & 0.0524 & 0.0274 & 0.0275 & 0.0273 \\
\hline & $\hat{\rho}_{12}$ & $\hat{\rho}_{13}$ & $\hat{\rho}_{14}$ & $\hat{\rho}_{23}$ & $\hat{\rho}_{24}$ & $\hat{\rho}_{34}$ \\
\cline { 2 - 7 } & 0.6888 & 0.6167 & 0.7270 & 0.6745 & 0.7724 & 0.9296 \\
Value & 0.0433 & 0.0495 & 0.0374 & 0.0445 & 0.0322 & 0.0112 \\
\hline sd & & & & & &
\end{tabular}


Table 5 Parameter values for each simulated scenario

\begin{tabular}{|c|c|c|c|c|c|c|c|c|}
\hline \multirow[b]{2}{*}{ Scenarios } & \multirow[b]{2}{*}{ Situation } & \multicolumn{7}{|c|}{ Parameters } \\
\hline & & $\phi_{1}$ & $\phi_{2}$ & $\nu_{1}$ & $\nu_{2}$ & $\sigma_{1}$ & $\sigma_{2}$ & $\rho_{12}$ \\
\hline 1 & $\begin{array}{l}\text { Less smoothness } \\
\text { and less variability }\end{array}$ & 0.05 & 0.1 & 0.3 & 0.4 & 0.5 & 1.0 & -0.7 \\
\hline 2 & $\begin{array}{l}\text { Less smoothness and } \\
\text { greater variability }\end{array}$ & 0.05 & 0.1 & 0.3 & 0.4 & 1.5 & 2.0 & 0.0 \\
\hline 3 & $\begin{array}{l}\text { Greater smoothness } \\
\text { and less variability }\end{array}$ & 0.05 & 0.1 & 0.7 & 1.0 & 0.5 & 1.0 & 0.7 \\
\hline
\end{tabular}

$\sigma_{2}^{2}=1.0$. Also in this scenario, we consider smaller smoothness for the variables, with values equal to $\nu_{1}=0.3$ and $\nu_{2}=0.4$. When $\nu=0.5$ the Matérn correlation function reduces to the exponential correlation function. Thus, this scenario represents lesser smoothness marginal behavior for the variables when compared to the exponential correlation model.

Scenario 2 keeps $\nu_{1}=0.3$ and $\nu_{2}=0.4$, and considers a greater variability with variances values equal to $\sigma_{1}^{2}=2.25$ and $\sigma_{2}^{2}=4.00$.

Scenario 3 considers a situation of smaller variability, with variance values fixed at $\sigma_{1}^{2}=0.25$ and $\sigma_{2}^{2}=1.0$, and considers higher smoothness values for variables: $\nu_{1}=0.7$ and $\nu_{2}=1.0$. In this scenario, we have marginal behaviors that are smoother in comparison with the exponential correlation model.

In all scenarios, we set the scale parameters values in $\phi_{1}=0.05$ and $\phi_{2}=0.1$. For the correlation parameter between the variables the values considered are $\rho_{12}=(-0.7,-0.4,0.0,0.4,0.7)$, aiming to illustrate different correlation structures that could occur in practice between the variables, with values ranging from a strong negative correlation $\left(\rho_{12}=-0.7\right)$ to a strong positive correlation $\left(\rho_{12}=0.7\right)$ and including the no correlation case where $\rho_{12}=0$.

For each scenario, 500 samples of a bivariate stationary isotropic Gaussian random field, of sizes 100, 225, 400 and 625, for each variable, were simulated in a regular unit grid. Fig. 7 illustrates the results of the simulated scenarios showing the expected bias plus and minus the expected standard error for estimators of the model for each scenario.

To facilitate the visualization we follow Bonat and Jørgensen (2016) and Petterle et al (2019), considering, for each parameter, standardized scales with respect to the standard error of the sample size 100, that is, for each parameter, the expected bias and the limits of the confidence intervals are divided by the standard error obtained on the sample of size 100. Standard errors and biases gets closer to zero as the sample size increases for the considered scenarios. In all scenarios, there appears to be a small overestimate for the smoothness and scale parameters, especially for smaller samples. 
For the $\mathrm{R}$ implementation, we use the Matrix package (Bates and Maechler, 2021) which provides efficient linear algebra calculations and the geoR package (Ribeiro Jr et al, 2020) for the Matérn correlation function. The mvnfast package (Fasiolo, 2016) was used for the simulation of the Gaussian process.

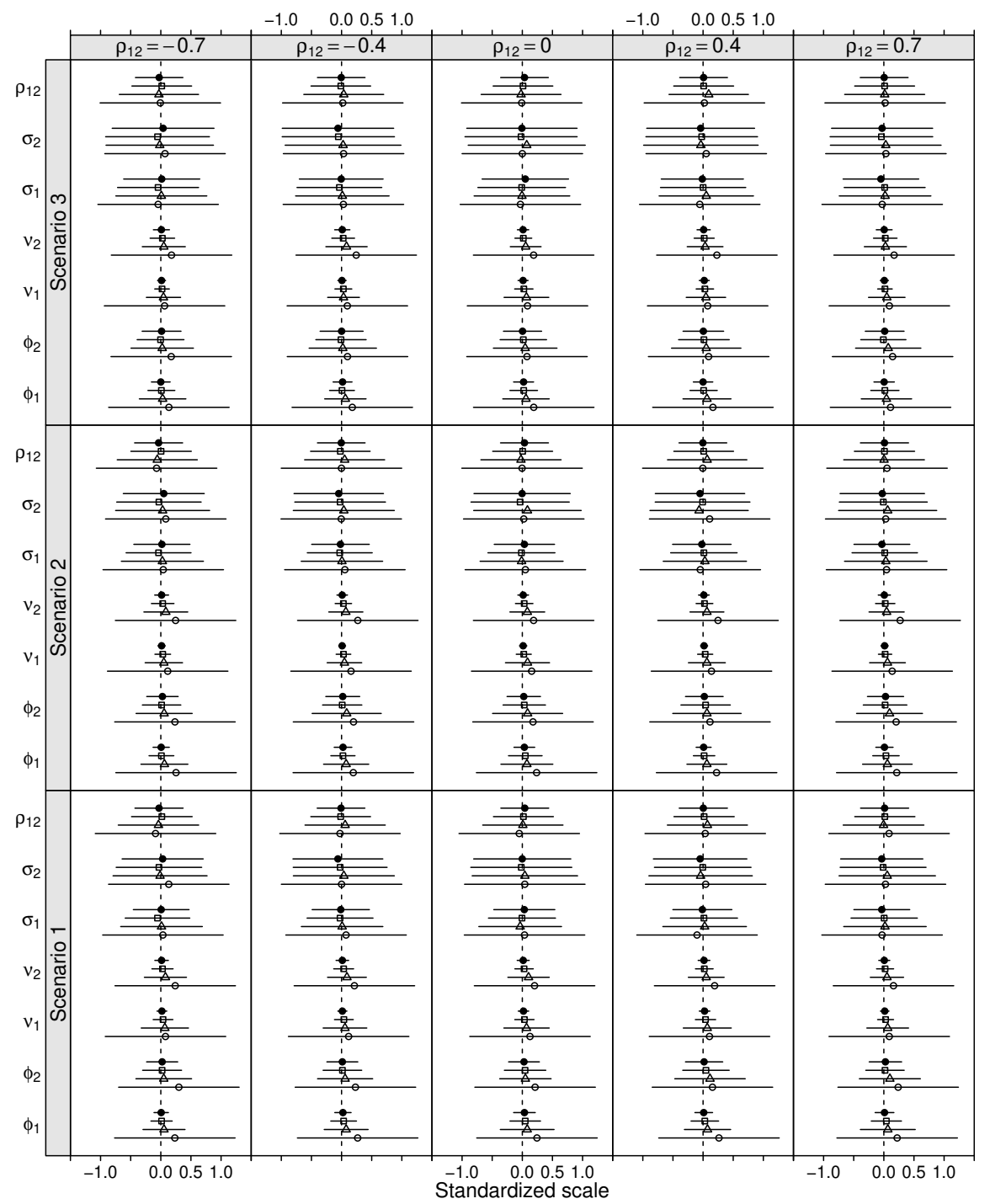

Fig. 7 Expected bias and confidence interval on a standardized scale for each scenario and sample size $(\bigcirc, 100 ; \triangle, 225 ; \square, 400 ; \bullet, 625)$ for the parameters of the MatSimpler model 


\section{Discussion}

We presented a covariance structure for multivariate random fields given by the product of matrices for continuously indexed data. The model is simple whilst flexible, allowing for different correlation structures and marginal-covariance functions. Its structure facilitates the specification, estimation, computation and generalization for more than two variables.

The analysis of the two data-sets illustrate the ability to deal with different covariance structures, allowing for different variability, smoothness and scales parameters. It also highlights the reduced computational times in comparison with other models. A simulation study with different combinations of model parameters suggests maximum likelihood estimators are asymptotically unbiased and consistent.

The major computational burden is the Cholesky decomposition, depending on the number of sample locations and response variables considered in the analysis. We illustrate the growth of computational time for up to six response variables and 900 sample locations. In this scenario, estimation time was approximately six hours (Fig. 8). Precise times will be hardware dependent. However, comparing it with some literature models for the bivariate case (Fig. 9), our proposal was competitive, presenting smaller estimation times, specially when compared to the MatConstr model, which has the same number of parameters.

Future directions include the study of estimation methods for the proposed approach that are more efficient, so that the estimation times will not be so penalized by the increase in variables and sampling locations. The organization and construction of an $\mathrm{R}$ package that involves the proposed approach shall be considered. Furthermore, the presented proposal opens options for future research in the context of non-Gaussian modeling and asymmetric data for multivariate spatial problems.

Supplementary materials. Supplementary materials for this article are available at: https://angelicamariatortola.github.io/academic/ MatSimpler2021.html.

\section{A Appendix. Computational results}

\section{References}

Alegría A, Porcu E, Furrer R (2018) Asymmetric matrix-valued covariances for multivariate random fields on spheres. Journal of Statistical Computation and Simulation 88(10):1850-1862. https://doi.org/10.1080/00949655.2017. 1406488

Alegría A, Porcu E, Furrer R, et al (2019) Covariance functions for multivariate gaussian fields evolving temporally over planet earth. Stochastic Environmental Research and Risk Assessment 33(8):1593-1608. https://doi.org/10. 


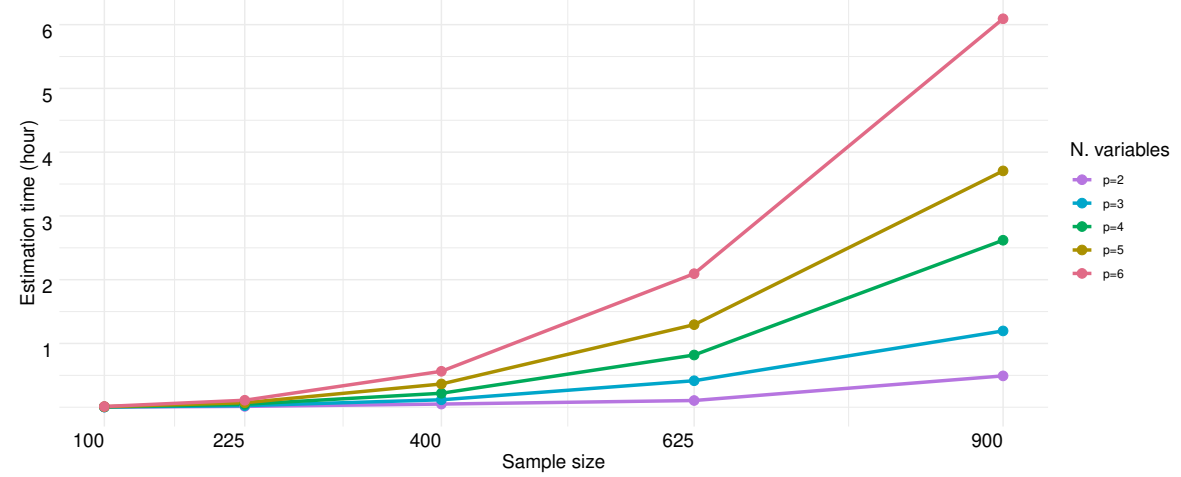

Fig. 8 Estimation time for MatSimpler model considering different sample sizes and number of variables

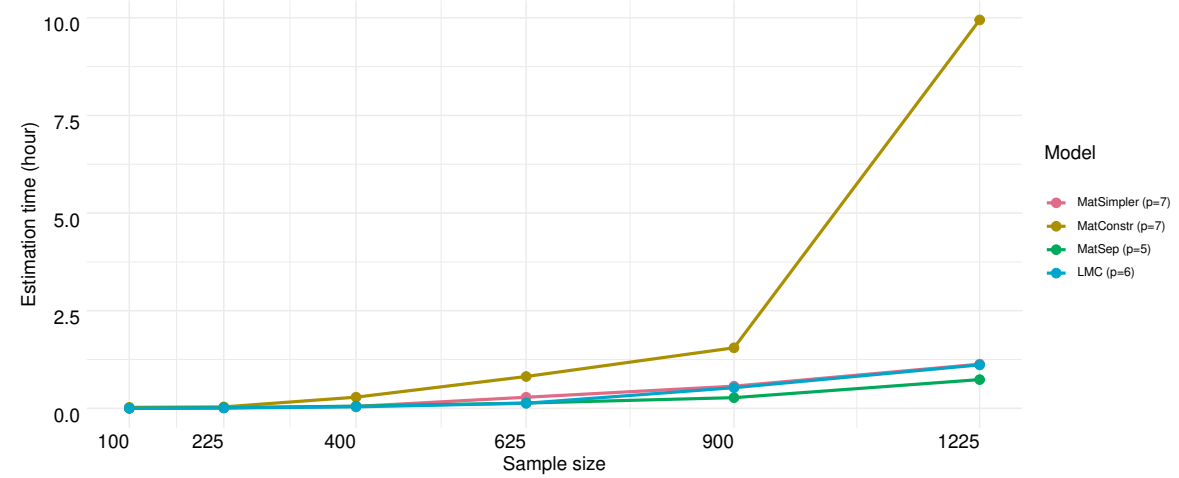

Fig. 9 Estimation times for the MatSimpler, MatConstr, MatSep and LMC models, considering simulated data from the MatConstr model for different sample sizes

1007/s00477-019-01707-w

Banerjee S, Roy A (2014) Linear algebra and matrix analysis for statistics. CRC Press, Boca Raton, https://doi.org/10.1201/b17040

Banerjee S, Carlin BP, Gelfand AE (2003) Hierarchical modeling and analysis for spatial data. Chapman and Hall/CRC, Boca Raton, https://doi.org/10. $1201 / 9780203487808$

Bates D, Maechler M (2021) Matrix: Sparse and Dense Matrix Classes and Methods. URL https://CRAN.R-project.org/package=Matrix, R package version $1.3-4$ 
Bevilacqua M, Morales-Oñate V (2018) GeoModels: A Package for Geostatistical Gaussian and non Gaussian Data Analysis. URL https://vmoprojs. github.io/GeoModels-page/, R package version 1.0.3-4

Bevilacqua M, Vallejos R, Velandia D (2015) Assessing the significance of the correlation between the components of a bivariate gaussian random field. Environmetrics 26(8):545-556. https://doi.org/10.1002/env.2367

Bevilacqua M, Alegría A, Velandia D, et al (2016a) Composite likelihood inference for multivariate gaussian random fields. Journal of agricultural, biological, and environmental statistics 21(3):448-469. https://doi.org/10. 1007/s13253-016-0256-3

Bevilacqua M, Fassò A, Gaetan C, et al (2016b) Covariance tapering for multivariate gaussian random fields estimation. Statistical Methods \& Applications 25(1):21-37. https://doi.org/10.1007/s10260-015-0338-3

Bevilacqua M, Diggle P, Porcu E (2020) Families of covariance functions for bivariate random fields on spheres. Spatial Statistics 40(100448):1-29. https: //doi.org/10.1016/j.spasta.2020.100448

Bivand RS, Pebesma EJ, Gómez-Rubio V, et al (2008) Applied spatial data analysis with R. Springer, New York, https://doi.org/10.1007/ 978-0-387-78171-6

Bonat WH, Jørgensen B (2016) Multivariate covariance generalized linear models. Journal of the Royal Statistical Society Series C (Applied Statistics) 65(5):649-675. https://doi.org/10.1111/rssc.12145

Bonat WH, Petterle RR, Balbinot P, et al (2020) Modelling multiple outcomes in repeated measures studies: Comparing aesthetic eyelid surgery techniques. Statistical Modelling 21:564-582. https://doi.org/10. $1177 / 1471082 X 20943312$

Bourgault G, Marcotte D (1991) Multivariable variogram and its application to the linear model of coregionalization. Mathematical Geology 23(7):899-928. https://doi.org/10.1007/BF02066732

Cecconi L, Grisotto L, Catelan D, et al (2016) Preferential sampling and bayesian geostatistics: Statistical modeling and examples. Statistical methods in medical research 25(4):1224-1243. https://doi.org/10.1177/ 0962280216660409

Chilès JP, Delfiner P (2012) Geostatistics: Modeling Spatial Uncertainty. Wiley, New York, https://doi.org/10.1007/s11004-012-9429-y 
Cressie N (1993) Statistics for spatial data. Wiley, New York, https://doi.org/ $10.1002 / 9781119115151$

Diggle P, Ribeiro Jr PJ (2007) Model-based Geostatistics. Springer, New York, https://doi.org/10.1007/978-0-387-48536-2

Emery X, Porcu E (2019) Simulating isotropic vector-valued gaussian random fields on the sphere through finite harmonics approximations. Stochastic Environmental Research and Risk Assessment 33(8):1659-1667. https://doi. org/10.1007/s00477-019-01717-8

Emery X, Porcu E, Bissiri PG (2019) A semiparametric class of axially symmetric random fields on the sphere. Stochastic Environmental Research and Risk Assessment 33(10):1863-1874. https://doi.org/10.1007/ s00477-019-01725-8

Fasiolo M (2016) An introduction to mvnfast. URL https://CRAN.R-project. org $/$ package $=$ mvnfast, $\mathrm{R}$ package version 0.1.6.

Finley AO, Banerjee S, Gelfand AE (2015) spBayes for large univariate and multivariate point-referenced spatio-temporal data models. Journal of Statistical Software, Articles 63(13):1-28. https://doi.org/10.18637/jss.v063. i13

Gelfand AE, Schmidt AM, Banerjee S, et al (2004) Nonstationary multivariate process modeling through spatially varying coregionalization. Test 13(2):263-312. https://doi.org/10.1007/BF02595775

Gentle JE (2017) Matrix algebra: Theory, Computations, and Applications in Statistics. Springer, New York, https://doi.org/10.1007/978-3-319-64867-5

Genton MG, Kleiber W (2015) Cross-covariance functions for multivariate geostatistics. Statistical Science 30(2):147-163. https://doi.org/10.1214/ 14-STS487

Gneiting T (1999) Correlation functions for atmospheric data analysis. Quarterly Journal of the Royal Meteorological Society 125(559):2449-2464. https: //doi.org/10.1002/qj.49712555906

Gneiting T, Kleiber W, Schlather M (2010) Matérn cross-covariance functions for multivariate random fields. Journal of the American Statistical Association 105(491):1167-1177. https://doi.org/10.1198/jasa.2010.tm09420

Goovaerts P, et al (1997) Geostatistics for natural resources evaluation. Oxford University Press, New York 
Goulard M, Voltz M (1992) Linear coregionalization model: tools for estimation and choice of cross-variogram matrix. Mathematical Geology 24(3):269-286. https://doi.org/10.1007/BF00893750

Guttorp P, Gneiting T (2006) Studies in the history of probability and statistics XLIX on the matérn correlation family. Biometrika 93(4):989-995

Hardy Y, Steeb WH (2019) Matrix Calculus, Kronecker Product and Tensor Product: A Practical Approach to Linear Algebra, Multilinear Algebra and Tensor Calculus with Software Implementations. World Scientific, Singapore, https://doi.org/10.1142/11338

MacNab YC (2016) Linear models of coregionalization for multivariate lattice data: Order-dependent and order-free cMCARs. Statistical methods in medical research 25(4):1118-1144. https://doi.org/10.1177/0962280216660419

MacNab YC (2018) Some recent work on multivariate gaussian markov random fields. Test 27(3):497-541. https://doi.org/10.1007/s11749-018-0605-3

Martinez-Beneito MA (2013) A general modelling framework for multivariate disease mapping. Biometrika 100(3):539-553. https://doi.org/10.1093/ biomet/ast023

Martinez-Beneito MA (2020) Some links between conditional and coregionalized multivariate gaussian markov random fields. Spatial Statistics 40(100383):1-17. https://doi.org/10.1016/j.spasta.2019.100383

Martins ABT, Bonat WH, Ribeiro Jr PJ (2016) Likelihood analysis for a class of spatial geostatistical compositional models. Spatial Statistics 17:121-130. https://doi.org/10.1016/j.spasta.2016.06.008

Matérn B (1986) Spatial variation. Springer, Berlin, https://doi.org/10.1002/ bimj.4710300514

Pebesma EJ (2004) Multivariable geostatistics in S: the gstat package. Computers \& Geosciences 30:683-691. https://doi.org/10.1016/j.cageo.2004.03. 012

Petersen KB, Pedersen MS, et al (2008) The matrix cookbook. Technical University of Denmark

Petterle RR, Bonat WH, Scarpin CT (2019) Quasi-beta longitudinal regression model applied to water quality index data. Journal of Agricultural, Biological and Environmental Statistics 24(2):346-368. https://doi.org/10. 1007/s13253-019-00360-8 
Porcu E, Daley DJ, Buhmann M, et al (2013) Radial basis functions with compact support for multivariate geostatistics. Stochastic environmental research and risk assessment 27(4):909-922. https://doi.org/10.1007/ s00477-012-0656-z

Qadir GA, Euán C, Sun Y (2021) Flexible modeling of variable asymmetries in cross-covariance functions for multivariate random fields. Journal of Agricultural, Biological and Environmental Statistics 26(1):1-22. https: //doi.org/10.1007/s13253-020-00414-2

R Core Team (2021) R: A Language and Environment for Statistical Computing. R Foundation for Statistical Computing, Vienna, Austria, URL https://www.R-project.org/

Ribeiro AMT, Ribeiro Jr PJ, Bonat WH (2021) Comparison of exponential covariance functions for bivariate geostatistical data. Revista Brasileira de Biometria 39(1):89-102. https://doi.org/10.28951/rbb.v39i1.558

Ribeiro Jr PJ, Diggle PJ, Schlather M, et al (2020) geoR: Analysis of Geostatistical Data. URL https://CRAN.R-project.org/package=geoR, R package version $1.8-1$

Rue H, Held L (2005) Gaussian Markov random fields: theory and applications. CRC press, Boca Raton, https://doi.org/10.1201/9780203492024

Salvaña MLO, Genton MG (2020) Nonstationary cross-covariance functions for multivariate spatio-temporal random fields. Spatial Statistics 37(100411):124. https://doi.org/10.1016/j.spasta.2020.100411

Särkkä S (2013) Bayesian filtering and smoothing. Cambridge University Press, Cambridge, https://doi.org/10.1017/CBO9781139344203

Schmidt AM, Gelfand AE (2003) A bayesian coregionalization approach for multivariate pollutant data. Journal of geophysical research 108(D24):1-9. https://doi.org/10.1029/2002JD002905

Teichmann J, Menzel P, Heinig T, et al (2021) Modeling and fitting of three-dimensional mineral microstructures by multinary random fields. Mathematical Geosciences 53(5):877-904. https://doi.org/10.1007/ s11004-020-09871-4

Vallejos R, Osorio F, Bevilacqua M (2020) Spatial relationships between two georeferenced variables: With applications in R. Springer, New York, https: //doi.org/10.1007/978-3-030-56681-4

Ver Hoef JM, Cressie N (1993) Multivariable spatial prediction. Mathematical Geology 25(2):219-240. https://doi.org/10.1007/BF00893273 
Wackernagel H (2003) Multivariate geostatistics: an introduction with applications. Springer, Berlin, https://doi.org/10.1007/978-3-662-05294-5

Wand M (2002) Vector differential calculus in statistics. The American Statistician 56(1):55-62. https://doi.org/10.1198/000313002753631376

\section{Statements and Declarations.}

- Funding: The authors declare that no funds, grants, or other support were received during the preparation of this manuscript.

- Competing Interests: The authors have no relevant financial or nonfinancial interests to disclose.

- Author Contributions: All authors contributed equally to the design and preparation of the material, with comments and suggestions for improvements. All authors read and approved the final manuscript. 